\title{
Amoebic peritonitis in pregnancy in the United Kingdom
}

\author{
G. Constantine, V. Menon and D. Luesley \\ Department of Obstetrics and Gynaecology, Dudley Road Hospital, Dudley Road, Birmingham, B18 7QH, UK.
}

\begin{abstract}
Summary: We describe a woman who developed hepatic amoebic abscesses during pregnancy, and subsequently amoebic peritonitis. We believe this to be the first such recorded case in the United Kingdom, and unusual in that the patient had never been abroad.
\end{abstract}

\section{Introduction}

Although often considered a disease of tropical and subtropical countries, amoebiasis does occur elsewhere. Whilst the patient has usually travelled abroad in the past, this need not always be the case. Pregnancy lowers immunity to many parasitic infections including amoebiasis, and thus patients with sub-clinical infestations may develop clinical symptoms during pregnancy as described in this case.

\section{Case report}

A 22 year old white woman booked at 13 weeks gestation in her second pregnancy. General examination was normal as were all booking investigations.

On review at 28 weeks static weight was noted, but all was otherwise normal. By 32 weeks gestation there had been no maternal weight gain, and clinical intrauterine growth retardation, confirmed by ultrasound scan, was noted. One week later, the patient was admitted as an emergency complaining of right sided pleuritic chest pain associated with dyspnoea and tachypnoea. She was apyrexial with a sinus tachycardia, the white cell count was $10.3 \times 10^{9} / 1$, and a chest X-ray revealed slight elevation of the right hemidiaphragm. No definitive diagnosis was made, and her symptoms resolved over the next 24 hours.

On the fourth day after admission she began vomiting and passing blood-stained diarrhoea. A gastroenterological opinion suggested that she had Crohn's disease, and treatment was commenced with steroid enemas. Her condition stabilized, but she continued to pass blood rectally and was started on ACTH injections. At no point was she noted to be pyrexial or clinically septicaemic. On the fifth day after admission she developed generalized abdominal tenderness and went into spontaneous labour. An

Correspondence: G. Constantine, M.R.C.P., M.R.C.O.G. Accepted: 16 December 1986 emergency caesarean section was performed because of a transverse lie.

A live infant weighing $1.92 \mathrm{~kg}$ was delivered. At operation, pus was noted in the right paracolic gutter, related to two hepatic abscesses in the right lobe of the liver, and the colon was dilated and inflamed. A defunctioning caecostomy was performed and the liver abscesses drained. The rectum appeared macroscopically normal on sigmoidoscopy, and a biopsy was taken. Post-operatively gentamicin and metronidazole were commenced. The presumptive diagnosis remained one of inflammatory bowel disease with secondary liver abscesses.

The patient remained stable over the next 24 hours but then developed pulmonary oedema, cyanosis, oliguria and shock. With intensive care an improvement was noted such that she was weaned off ventilator and inotropic support 10 days after delivery.

Microscopy of the pus and rectal biopsies revealed the presence of Entamoeba histolytica, although no organism was cultured, and metronidazole was thus continued. On the 47th day after admission and 42 days after delivery, she was discharged home.

\section{Discussion}

In the United Kingdom it is estimated that there are about 3 deaths and 200 new cases of amoebiasis each year. ${ }^{1}$ Cases of hepatic amoebiasis described in this country have been virtually all in men, many, but not all of whom had been abroad at some time in their life. ${ }^{2,3}$ Some patients had never been abroad and others were thought to have contracted the disease from relatives. ${ }^{4}$

The patient described above is unusual in that she had never been abroad and neither had any relative. When she acquired the condition is unknown as amoebiasis can remain quiescent for many years. It must however have been in the United Kingdom. 
Amoebiasis is known to be more severe in pregnant women. ${ }^{5,6,7}$ This is thought to be due to raised progesterone levels ${ }^{8}$ and failure of immunoglobulin levels to rise in infection. ${ }^{9}$ In our case immunoglobulin levels were within the normal range.

Amoebic dysentery in pregnancy is extensively reported, amoebic liver abscesses less so. Cases previously reported have all been from areas where the disease might be expected to be relatively common. ${ }^{10-17}$ We believe our patient to be the first reported in pregnancy in the United Kingdom.

In virtually all of the above cases, as in our case, misdiagnosis occurred, and in many the diagnosis was made at laparotomy. Intestinal involvement is by no means universal in hepatic amoebiasis, as $30-50 \%$ of patients with liver abscesses give no bowel related history. ${ }^{6,18}$ In our case some, but not all, of the classic features of hepatic amoebiasis were present. This suggests the need for a high index of suspicion when patients present with right sided chest pains and tenderness, or weight loss, even if they are apyrexial and have no bowel related symptoms.

This case illustrates once again the need to exclude positively an infestation/infection before diagnosing Crohn's disease or ulcerative colitis and instituting

\section{References}

1. Stamm, W.P. Amoebiasis in England and Wales. Br Med $J$ 1975, 1: 452-454.

2. Paulley, J.W. Amoebic liver abscess in Great Britain. Br Med J 1961, 1: 462.

3. Wright, R. Amoebiasis - a diagnostic problem in Great Britain. Br Med J 1966, 1: 957.

4. Brown, R.C., McKendrick, M.W., Grainger, K. \& Paulley, J.W. Amoebic liver abscess - potential causes of delay in diagnosis. Postgrad Med J 1978, 54: 555-558.

5. Abioye, A.A. Fatal amoebic colitis in pregnancy and the puerperium. J Trop Med Hyg 1973, 76: 97-100.

6. Abioye, A.A. \& Edington, G.M. Prevalence of amoebiasis at autopsy in Ibadan. Trans $R$ Soc Trop Med Hyg 1972, 66: 754-763.

7. Lewis, E.A. \& Antia, A.U. Amoebic colitis: review of 295 cases. Trans R Soc Trop Med Hyg 1969, 63: 633.

8. Biagi, F.F. \& Beltran, H.F. The challenge of amoebiasis: understanding pathogenic mechanisms. Int Rev Trop Med 1969, 3: 219-239.

9. Abioye, A.A., Lewis, E.A. \& McFarlane, H. Clinical evaluation of serum immunoglobulins in amoebiasis. Immunology 1972, 23: 937-946.

10. Cowan, D.B. \& Houlton, M.C.C. Rupture of an amoebic liver abscess in pregnancy. $S$ Afr Med $J 1978$, 53: 460-461.

11. De Silva, K. Intraperitoneal rupture of an amoebic liver treatment for these.

The combination of severe amoebic colitis, together with a ruptured hepatic abscess appears unusual in the cases described previously in pregnancy. This may have contributed to our patient's stormy postoperative recovery when compared with previous cases described in pregnancy. In non-pregnant patients with ruptured hepatic abscesses, an overall mortality of $42 \%$, increasing with age and delayed diagnosis, is recorded. ${ }^{12}$ Partially due to the debilitated state of the patients, post-operative morbidity is high and should be anticipated. Cardiovascular and respiratory problems, metabolic disturbances, renal failure and shock are all described, and may need specialist support and treatment.

In uncomplicated amoebic dysentery and small liver abscesses, metronidazole alone may be effective. In larger unruptured abscesses trans-abdominal aspiration may be attempted. If rupture is suspected, laparotomy is essential and lifesaving. . $^{12,19}$

We hope that our experience highlights the possibility of patients acquiring and developing hepatic amoebiasis in this country, the particular dangers of pregnancy, and the potentially life-threatening consequences if diagnosis and treatment is delayed.

abscess in a pregnant women at term. Ceylon Med J 1970 15: $51-53$.

12. Eggleston, F.C., Handa, A.K. \& Verghese, M. Amoebic peritonitis secondary to amoebic liver abscess. Surgery 1982, 91: 46-48.

13. Mitchell,L.W. \& Teare, A.J. Amoebic liver abscess in pregnancy. Case reports. Br J Obstet Gynaecol 1984, 91: 393-395.

14. Naidoo, P.M., Keeton, G., Stein, L., Kottler, R. \& Horwitz, M. Hepatic amoebiasis - a study of 32 cases in the Western Cape. S Afr Med J 1974, 48: 1159-1160.

15. Navaratne, R.A. Postpartum intraperitoneal rupture of an amoebic liver abscess. Ceylon Med J 1972, 17: 160163.

16. Wagner, V.P., Smale, L.E. \& Lischke, J.H. Amoebic liver abscess of the liver and spleen in pregnancy and the puerperium. Obstet Gynecol 1975, 45: 562-565.

17. Yen. S.S. Spontaneous rupture of the liver during pregnancy - a report of two cases. Obstet Gynecol 1964, 23: 783-787.

18. Barrett-Connor, E. Amoebiasis today in the United States. Calif Med 1971, 114: 1-6.

19. Licad, J.V. \& Recio, P.M. Clinical features, complications and surgical treatment of amoebic hepatic abscess a study of 120 cases. Int Surg 1969, 51: 183-189. 